\title{
Fuel R\&D Needs and Strategy towards a Revision of Acceptance Criteria
}

\author{
François Barré, Claude Grandjean, Marc Petit, and Jean-Claude Micaelli \\ Institut de Radioprotection et de Sûreté Nucléaire (IRSN), Direction de Prévention des Accidents Majeurs, BP3, \\ 13115 Saint-Paul lez Durance Cedex, France \\ Correspondence should be addressed to François Barré, francois.barre@irsn.fr
}

Received 5 May 2009; Accepted 30 December 2009

Academic Editor: Nikola Čavlina

Copyright ( 92010 François Barré et al. This is an open access article distributed under the Creative Commons Attribution License, which permits unrestricted use, distribution, and reproduction in any medium, provided the original work is properly cited.

The study of fuel behaviour under accidental conditions is a major concern in the safety analysis of the Pressurised Water Reactors. The consequences of Design Basis Accidents, such as Loss of Coolant Accident and Reactivity Initiated Accident, have to be quantified in comparison to the safety criteria. Those criteria have been established in the 1970s on the basis of experiments performed with fresh or low irradiated fuel. Starting in the 1990s, the increased industrial competition and constraints led utilities to use fuel in more and more aggressive conditions (higher discharge burnup, higher power, load follow, etc.) and create incentive conditions for the development of advanced fuel designs with improved performance (new fuel types with additives, cladding material with better resistance to corrosion, etc.). These long anticipated developments involved the need for new investigations of irradiated fuel behaviour in order to check the adequacy of the current criteria, evaluate the safety margins, provide new technical bases for modelling and allow an evolution of these criteria. Such an evolution is presently under discussion in France and several other countries, in view of a revision in the next coming years. For this purpose, a R\&D strategy has been defined at IRSN.

\section{Industrial Context}

The economic context is increasingly demanding for the utilities who, in order to keep competitive, have to produce the cheapest possible KWh while retaining sufficient flexibility to adjust the energy produced to the demand. The response of nuclear reactor operators is to increase reactor power, the cycle length (and thus discharge burnup), and the fuel enrichment. Applying flexible operating modes involve power variations imposing high stresses on the fuel which must be taken into account when considering changes in Safety Assessment.

Fuel rods are therefore subjected to increasing mechanical, thermal and chemical stresses in the new fuel management procedures, while having to satisfy the following contradictory requirements:

(i) reliability, to avoid degrading the operating conditions (e.g., few manufacturing defects, low deformation of assemblies, etc.),

(ii) robustness, to withstand the contingencies of the operating conditions (e.g., low corrosion), (iii) high performance, to provide margins that can be used in operation (sufficiently high PCI limit; proper behaviour in accident conditions: RIA, LOCA, etc.).

Furthermore, fuel elements are complex objects, the design of which requires an in-depth knowledge of coupled phenomena (neutronics, thermohydraulic, thermomechanical, chemical) that are very difficult to separate out. To ensure the reliability and robustness of a new fuel element, to predict its performance (in particular, from a safety point of view, its behaviour in accidental conditions), it is therefore necessary to make use of simulation duly validated by experiment, including integral tests which may reveal previously unknown phenomena.

In addition, the number of fuel rods under operation in the world is very high and the probability to have defect rods or unknown phenomena is nonnegligible. For example, in France, the utility EDF manages more than 10,000 irradiated assemblies, representing about 2.7 million fuel rods, which are loaded in NPP reactor cores, exposed to a harsh environment (high temperature: $300^{\circ} \mathrm{C}$ for the 
coolant, high flow velocity, aggressive chemistry: borated, lithiated fluid) over periods of up to six years.

In the current increasingly competitive context, the utilities clearly indicate that, for PWR presently under operation, there is a constant need to improve fuel performance and develop innovative products for a more efficient use of fuel at a time when the uranium market will be under pressure, so as to minimize the quantity of waste, to increase unit power and cycle length, and so forth.

It is planned to reinforce $\mathrm{R} \& \mathrm{D}$ on fuel for a continued improvement of the present products and optimization of their management and, in the long term, to design new fuel elements that represent a real break with today's current concepts. Substantial evolution of present technologies has been developed for an increase in robustness and performance, for example, a constant upgrading of the fuel matrices (UO2, MOX, etc.), a general use of consumable poisons, the introduction of doped pellets, and also continuous cladding evolution, with the introduction of M5, optimized ZIRLO, MDA, etc. New generation of products for the PWRs is under preparation.

Moreover, all operators are going to both diversify their fuel supply by calling upon manufacturers that are developing different technologies and benefit from progress made in fuel element design.

Thus, the diversity in fuel supply and in the operation of the various units means that a very large number of initial states and responses to various stresses in incident and accident conditions have to be taken into account in accident studies, the evolution of acceptance criteria, and available methodologies for safety demonstration.

\section{Safety Needs}

Safety demonstrations for pressurised water reactors are deterministic, supplemented by a probabilistic approach. The deterministic method is based on analysing postulated initiating events, which can be grouped to define a limited number of operating transients and design-basis incidents and accidents, which constitute the design-basis operating conditions. The studies of design-basis operating conditions must demonstrate compliance with safety requirements involving the first safety barrier that is the fuel cladding. As part of the safety demonstration, the safety requirements are developed into decoupling criteria (improperly referred to as safety acceptance criteria).

The objective is to guarantee a proper behaviour of the first barrier, in all conditions, including extended periods of operation, incidental situations, low-probability extreme accident conditions such as loss-of-coolant accidents (LOCAs) or reactivity-initiated accidents (RIAs). For such conditions the utility has to demonstrate the possibility of keeping the nuclear reaction under control, ensuring the short-term and long-term core cooling and a limited extend of cladding degradation, this last one being the first safety barrier.

In this respect, a safety baseline is used which defines the fuel operating conditions, the phenomena to be taken into account in normal operation or in incident or accident conditions, the computing methodologies and tools to be used for safety demonstration, and of course the acceptance criteria to be met. IRSN must make sure that this safety baseline is relevant (if necessary pressing for changes) and that the criteria are met, and must also assess the operating margins during reactor operation.

Expert assessment of fuel is an excessively difficult field, first because of the complexity of the physics (multidisciplinary), the mechanisms of which are difficult to separate out and subjected to very varied boundary conditions (large number of external stresses). The difficulties are also technological, because of a large variety of technologies (constantly evolving field), a growing diversity and change in the operating conditions (leading to diversity of changes in the fuel while it is in the reactor), and the diversity of incident and accident conditions to be taken into account over the whole downstream fuel cycle.

Furthermore, the utility, in its concern for economic profitability and therefore always getting the most out of the fuel, is led to use ever-more-sophisticated methods and arguments in order to demonstrate compliance with the safety baselines.

Thus Safety Assessment should be based on a well-thought-out simulation/experimentation R\&D strategy, including in-pile integral testing. The IRSN has to conduct such a major R\&D programme, so as to be in a position to validate products, assessment methodologies, and operating conditions. One reason is that Technical Support Organisation (TSO), such as IRSN, should be leader in the investigation of accidental situations. They must also develop generic knowledge and computing tools because of the diversity of situations that will have to be assessed (technologies, operation, etc.), have their own computing tools and methodologies in order to ensure the independence of their judgement, and provide high-level skills training for expert assessment.

In this paper we focus on the $R \& D$ needs towards a revision of acceptance criteria and the evolution of methodologies used for the safety assessment for LOCA and RIA.

\section{R\&D Strategy for Revisiting Loss of Coolant Accidents (LOCAs); The CYCLADES Project}

The LOCA results from a break in the primary circuit that leads to the depressurisation of the primary system with power decrease to its residual level and water reflooding. As a consequence, the core fuel rods undergo the following evolution: clad temperature increase and dry-out, transfer of the stored energy from the fuel to the clad, clad ballooning with possible contact with the neighbouring rods, burst failure around $800^{\circ} \mathrm{C}$ and fuel relocation inside the ballooned zone; beyond this phase, oxidation of the cladding will occur at high temperature leading to a significant clad embrittlement before quenching due to reflooding water.

The LOCA is a Design-basis accident (DBA) used for the design of the safety emergency cooling systems and the limitation of the power at the hottest point in the core during normal operation. The safety principle is that the coolability 
of the core has to be preserved. The associated safety requirements are to ensure the resistance of the fuel rods upon quench and postquench loads and to maintain a coolable geometry in the core. These requirements are formalised in safety criteria, associated with limit values, mainly expressed through a maximum cladding temperature (PCT) and an equivalent cladding reacted ratio (ECR) (ECR is the ratio of the cladding thickness equivalently transformed into $\mathrm{ZrO}_{2}$ by oxidation over the initial clad thickness, taking into account possible thinning during clad ballooning) with the aim of ensuring a residual ductility of the cladding for maintaining core coolability capability on short- and long-term duration. In France as in the U.S., the current limits are $1204^{\circ} \mathrm{C}$ on PCT and $17 \%$ on ECR. These LOCA acceptance criteria are issued from a long process in the United States (Ergen Task force in 1967, Interim Acceptance Criteria for ECCS in 1971, then ECCS Rule-making Hearing in 1972-1973). Since they were established, the evolution of fuel operating conditions and fuel technologies leads the international community to review the technical basis to reassess these criteria and their limit values.

In order to prepare acceptance criteria reassessment, IRSN recently conducted an extensive Start-of-the-ArtReview relative to fuel behaviour under LOCA conditions, covering the aspects of clad ballooning and flow blockage, coolability of partially blocked assemblies, clad oxidation, and clad resistance to quench and postquench loads [1-3]. Additionally, a review of existing computation tools devoted to the calculation of fuel behaviour under LOCA was performed. Together with the outcomes from the recent results from experimental program, this leads to summarising the main pending questions relative to fuel behaviour under LOCA in the three following topics:

(i) the cladding embrittlement, to address the loss of cladding integrity upon quench and postquench loads,

(ii) the relocation of fuel in the ballooning parts of the fuel rods,

(iii) the flow blockage by ballooning rods and its coolability.

IRSN considers important that these questions be solved to guarantee core coolability in a postulated LOCA transient and has launched an R\&D project, named CYLCADES, to investigate them.

3.1. Cladding Embrittlement. The effects of both alloy composition and burnup on cladding embrittlement were extensively studied [3,4] but questions still remain to properly understand physical phenomena and to ensure a correct prediction of the behaviour of future cladding alloys. Some of the hydrogen that is liberated in the corrosion process enters the cladding during normal operation. The hydrogen was found to produce a strong effect on the embrittlement of the cladding, but the effect is indirect. Hydrogen mainly acts as a catalyst while oxygen, which diffuses into the cladding metal during a LOCA transient, and is the direct cause of embrittlement. Oxygen from the oxide fuel pellets was found to enter the cladding from the inner side in high-burnup fuel in addition to the oxygen that enters from the oxide layer on the outer side of the cladding. Under conditions that might occur during a smallbreak LOCA, the accumulating oxide on the surface of the cladding can break up and was found to let large amounts of hydrogen into the cladding during the LOCA transient, thus exacerbating the embrittlement process. The current work also confirmed an older finding that, if rupture occurs during a LOCA transient, large amounts of hydrogen can enter the cladding from the inside near the rupture location.

Postquench ductility, a commonly used parameter in the past as the basis of safety criteria, appears as an inappropriate parameter to define an embrittlement threshold, since no practical limit can be derived to ensure ductility retention in the cladding balloon. However, the results from ANL 4point bending tests [5] at room temperature on unirradiated samples after integral testing suggest that, below some oxidation limit, the rod cladding would resist fragmentation when subjected to quench and postquench loads.

A strength-based approach addressing the structural response of the whole cladding, instead of a ductility approach, might provide an acceptable alternative. IRSN has proposed a two-step R\&D strategy to address those questions.

The first step is to quantify a relevant embrittlement threshold. The first important item is to identify and quantify mechanical loads that should be supported by fuel rods during quench and post-quench phases of LOCA transients. This will be informed through from numerical simulations in bundle geometry. It is a necessary challenge. Afterwards, appropriate structural response tests, taking into account bounding mechanical loads (quench under controlled loading, impact tests, etc.) to determine embrittlement threshold, should be defined. Finally, it is necessary to carry out Finite Element calculations with typical cladding geometry and properties to verify that the experimental domain covered by this type of tests is adequate. This step may require appropriate analytical experimental tests to characterise the evolution of clad properties, in particular at the location of the balloons.

The second step is to select the most appropriate parameter characterising the oxidation and hydriding amount on which to correlate embrittlement, so as to find out an alternative to the ECR. It is based on the previous work from early investigators ([6-9], etc.). At that time, research programs and calculation tools were not ready to make successful these attempts. Based on the one hand on this previous work and on the other hand on recent important research results obtained at ANL [4], CEA [10], or JAEA [11], IRSN considers that an embrittlement threshold based on residual thickness of prior $\beta$-Zr layer with low oxygen content as function of $\mathrm{H}$ content should be more appropriate. In this respect, IRSN has been developing an R\&D program which consists in a computer code development (diffusion code DIFFOX), with the support of specific tests and experimental techniques (IRSN MARGO-R tests), which makes possible to correctly evaluate the thickness of the $\beta$ layer and the oxygen distribution in this layer [12]. 
3.2. Axial Fuel Relocation. During normal operation, oxide fuel pellets develop many cracks because of thermal stresses. After clad ballooning and burst, some fragmented fuel particles located above the ballooned region of a fuel rod will thus relocate into the enlarged volume of the balloon under the influence of gravity and pressure differences. This effect was first noticed in 1980 in reactor tests in the United States (PBF/LOC tests, INEL, [13]), Germany (FR-2 tests, KFK, Karb 83), and France (FLASH-5 tests, Bruet 92), and recent integral tests at Argonne National Laboratory (ANL) and the Halden Reactor Project in Norway (IFA-650 test series) have confirmed it. The consequence of fuel relocation is an increase in heat generation in the ballooned region together with a reduction of the thermal resistance between fuel and cladding, which may increase the cladding temperature and oxidation compared with an undeformed length of the fuel rod.

The strategy proposed by the IRSN is to carry out integral tests with different cladding, different types of fuel pellets, and different burn-ups, in particular to actively support the on-going OECD program performed in the Halden reactor (IFA-650 test series) and to consider complementary tests in the Halden reactor or the Cabri reactor (with the use of the hodoscope which allows to analyse precisely the fuel relocation without moving the rods). A consistent and sound interpretation of this large range of older and recent tests has to be performed and will be supported by laboratory studies to understand the basic mechanisms of fuel fragments slumping and accumulation in a cladding balloon and then to model fuel fragmentation as function of fuel types, burn-up, and power load history. Then, in the calculation tools, balloon filling ratio should be given as a function of fuel rod characteristics.

\subsection{Flow Blockage and Its Coolability}

3.3.1. Flow Blockage Characteristics. The question of the cumulative effects of irradiation, which homogenize the azimuthal temperature distribution in the fuel rod cladding, and of the bundle size on the main characteristics of the blockage (maximum flow restriction ratio, axial extent) has been under investigation for a long time and several programmes have been devoted to it (see [1]). Based on a comparison of the burst strains obtained in ORNL Single rod/ Multirod tests and in PBF-LOC tests with fresh rods/irradiated rods, one of the major conclusions of the past R\&D programmes was provided by INEL, which recommended to perform in-pile bundle tests of sufficient bundle size with irradiated rods [13].

However, such a strategy might lead to significant costs; indeed, a short series of in-pile integral bundle tests could close the issue for a given fuel but let it open for another type of fuel; in addition, in-pile experimental facilities for testing with large size bundles are more and more difficult to find (PHEBUS reactor has been recently shutdown!).

A simulation strategy based on the use of a detailed and well assessed code is proposed by IRSN. For this purpose, with the support of EDF, IRSN is developing the DRACCAR code [14] which is a multirod $3 \mathrm{D}$ thermomechanics code, with mechanical and thermal interactions between rods, coupled with subchannel type two-phase flow codes. DRACCAR code has to be rigorously assessed prior to reactor applications. The model validation will be based on the results of existing experimental programmes and additional in-pile tests with single irradiated fuel rods in a well instrumented prototypical environment; these tests could be performed in the Cabri reactor and could possibly be complemented by "small" bundle tests. This can be supported, if necessary, by more analytical out-of-pile tests focusing on clad-to-clad thermomechanical interactions and balloon axial extension.

3.3.2. Flow Blockage Coolability. IRSN has carried out a stateof-the-art review of the main experimental programmes related to the question of the coolability of blocked regions in a rod bundle after ballooning in a LOCA, such as the FEBA, SEFLEX, THETIS, ACHILLES, CEGB, and FLECHTSEASET programmes [2], as well as of several analytical developments performed in association with these experimental programmes. The conclusions drawn from these results were used to improve our understanding of the physical phenomena governing the behaviour of a partially blocked rod array during a LOCA reflood scenario and to evaluate the limits of blockage coolability under the most severe geometric (blockage ratio and length) and thermohydraulic conditions. However, it is important to underline that these results were obtained in out-of-pile experiments performed with electrically heated fuel rod simulators with a large gap between the heaters and the ballooned cladding. These experiments promote the cladding coolability because it is separated from the heat source by a wide gap, which is not representative of the situation with fragmented fuel accumulated in the cladding balloons (fuel relocation), as was observed during all in-pile tests with irradiated fuel rods. The impact of fuel relocation upon blockage coolability therefore remains to be investigated.

It is necessary to perform out-of-pile experiments with a partially blocked full-length bundle, with realistic simulation of fuel relocation in the balloons, in view of updating the upper bound value of maximum blockage remaining coolable. It can be supported by some more analytical tests addressing basic phenomena (droplet impact and fragmentation, heat transfer on balloon walls, flow redistribution, etc.) to reach a better understanding of the leading phenomena and to select the most appropriate form of heat transfer correlations for the DRACCAR code.

\section{R\&D Strategy for Revisiting Reactivity Initiated Accident (RIA)}

The postulated reactivity initiated accident results from the failure of the drive mechanism of a control rod cluster made of absorber rods regulating the nuclear reaction. This failure causes the ejection of the control rod cluster due to pressure difference (around 150b) between reactor cooling system and containment and leads to reactivity insertion. This accident generates a fast power transient (in some tens 
of milliseconds) with significant energy injection in the fuel rods neighbouring the ejected control rod assembly, and raises the following questions.

(i) Do the fuel rods fail and what are the rod failure conditions for the various fuel types, operating conditions experienced by the fuel rod, and burnup levels?

(ii) Does ejection of solid fuel particles into the coolant occur after rod failure?

(iii) If fuel is ejected, could the fuel-coolant thermal interaction propagate the accident to the neighbouring rods and jeopardize the core cooling capability?

RIA is a DBA; the safety principle is the preservation of core coolability. The associated safety requirements are defined in order to limit the fuel pellets melting and the number of fuel rods affected by boiling crisis and to avoid fuel dispersion in the primary coolant. The associated current safety criteria for RIA have been formulated in the 1970s on the basis of the available experimental database (from SPERT, PBF, and early NSRR experiments) that was restricted to fresh or slightly irradiated UO2 fuels (up to $30 \mathrm{GWd} / \mathrm{tU}$ for UO2 fuel) and with cladding and fuel technologies which are no more currently in operation. However, the clear evidence of specific aspects of highly irradiated fuel that could affect the transient rod behaviour during a RIA and the lack of data on irradiated MOX fuel, created the need to both verify and/or adjust the current safety criteria and to evaluate the corresponding margins. As a result, various organizations in most countries operating reactors [15-17] are assessing the RIA limits that apply to current and future fuels, in terms of component materials as well as burn-up.

Indeed, several CABRI REP tests (Na-1 to Na-12) on $\mathrm{UO}_{2}$ and MOX rods with zircaloy-4 cladding were carried out by IRSN between 1993 and 2000 in the sodium loop of the CABRI reactor. The test results revealed rod failures for enthalpy values less than the SPERT failure threshold of $140 \mathrm{cal} / \mathrm{g}$ for irradiated fuels. These CABRI tests clearly showed the need to define new criteria for irradiated fuel [18]. Most countries that operate reactors decided that the RIA criteria were in need of changes and are cofinancing experimental programmes to generate fuel behaviour data. This primarily concerns the CIP programme (IRSN) [19] and the ALPS programme (JAEA) [20].

4.1. The IRSN Strategy. In order to be able to give relevant technical advice on revising the RIA criteria, the IRSN felt it is necessary to develop its own methodology for deriving RIA safety limits. Indeed, developing a methodology call for a thorough understanding of the physical mechanisms involved in each phase of an RIA, supported by interpretation of the experimental database, developing computing codes, identifying the relevant parameters using the above methodology. All those aspects are required for an accurate assessment of the safety criteria and methodologies proposed by the utilities.
Taking into account these considerations, as well as the continuous fuel evolutions, the complex and intimately coupled phenomena under transients, IRSN, develops an R\&D strategy which aims at getting physical bases for establishing new safety criteria. The approach consists of

(i) the development of the SCANAIR computational tool dedicated to the description of the global fuel rod behaviour in reactor conditions; it has to be validated on the available tests for valuable prediction capability and for use in supporting expertise studies,

(ii) the acquisition of the necessary knowledge and identification of the main phenomena, through inpile testing programs in which consequences of a fast power transient applied to an industrial irradiated fuel rod (single) can be studied under representative reactor conditions (nuclear heating, coolant temperature, thermo-mechanical loading, etc.) up to rod failure and postfailure events, taking into account the intimately coupled phenomena;

(iii) the performance of separate effect tests programs aiming at the back-up of the SCANAIR development and at improvement of the understanding of some parts of the phenomenology; for instance, the Prometra program for mechanical characterisation of the Zr-4 and advanced claddings Zirlo and M5 [21], the Patricia program for the study of clad to coolant heat transfer under fast transient [22], the fission gas dynamic (FGD) program under preparation within cooperation with JAEA;

(iv) the development of a more detailed modeling with a multiscale approach when possible, allowing confirmation of the necessarily simplified models implemented in the reactor computational tool.

4.2. In-Pile Experiments. The main outcomes of the Cabri REP-Na program, conducted by the IRSN in 1992-2002 with the support of EDF, was the role of hydrides in the risk of rupture, the contribution of fission gases at the grain boundaries, the importance of oxide layer spalling, and above all the need to update the current safety criteria, which are no longer appropriate for present operation conditions. The Cabri International Program conducted by IRSN, launched in 2002, under OECD auspices with a broad international cooperation and partnership of EDF, will be carried out in the renewed facility under typical pressurised water reactor conditions. The main objectives are to address the pending questions relative to the fuel rod behaviour over the whole transient, the occurrence of boiling crisis and its consequences on rod failure with high clad temperature, high coolant and rod internal pressures and fission gas loading, and the postfailure phenomena (fuel ejection, fuel-coolant interaction with finely fragmented solid fuel, thermal to mechanical energy conversion). The capability of the CABRI reactor with typical PWR conditions and its versatility will allow exploring the influence of the pulse width on the phenomena as well as the effect of advanced fuel microstructures for $\mathrm{UO} 2$ and MOX. 
Today the IRSN is moving towards "analytical" safety criteria $[23,24]$; implementation of which is based on more detailed understanding of the physical phenomena involved (e.g., the different clad failure modes of hydrided claddings). Same kinds of approaches are developed by EDF and EPRI [24]. Studies in progress at present, in particular at the IRSN, suggest that the study of the zero power RIA is not necessarily conservative for all cases and that conditions at intermediate power must be considered. The Cabri REP-Na and CIP0 programmes have shown that the tests were very complex and could not be used in a binary manner (failed/not failed). Their interpretation necessitates analytical tests, upstream research, use of complex simulation tools, and understanding of coupled basic phenomena (mechanics, materials, thermochemistry). No consensus has been reached on the interpretation of some tests in the REP-Na programme, and comparison with the NSSR tests rises up new questions, for example, the effect of initial test temperature. Lastly, the criteria as conceived for the binary failed/not failed approach would necessitate thorough RIA tests on all types of fuel, cladding, and burnup and having experienced all types of transient during life in a reactor, which is not achievable. A simulation approach must therefore be considered in order to extrapolate to actual rods under reactor conditions, but validated by a broader range of RIA tests and analytical tests. Changes in the safety baseline, like fuel technologies, lead inevitably to consideration of new programmes after CIP, taking up a clear position in favour of a simulation approach.

The objectives of future programmes in Cabri will be to validate the new analytical safety criteria, to test rods with new fuel rod technologies, to explore accident conditions not yet investigated.

Tests at zero initial power must therefore be considered, updating the concept (and the instrumentation) to give a better response to the following current approaches:

(i) more analytical tests, on lengths of moderate size prepared from a given rod stage, scanning physical parameters, for example, different deposited energies; tests on defective rods for more specific study of fuel ejection conditions and consequences might be considered;

(ii) tests to explore other types of pulse insofar as late injected energy could have a strong influence on behaviour after departure from nucleate boiling;

(iii) RIA tests similar to those of the CIP programme (full length in particular) on new fuel rods (changes in cladding, fuels, etc.); potential needs include changes in fuels (additives, pellet geometries), high burnup ( $>45 \mathrm{GWd} / \mathrm{t}$ ) gadolinium-containing rods, VVER fuels, new products to counter PCI (short pellets), and so forth.

In parallel, it is also necessary to explore the cases at intermediate powers which are not covered by the transients at zero power.

\section{Conclusion}

The increased industrial competition and constraints result in more aggressive conditions for the fuel (higher burnup, higher power, load follow, etc.) and create incentive conditions for the development of advanced fuel designs with improved performance (new fuel types with additives, cladding material with better resistance to corrosion, etc.). This situation involved the need for new investigations of irradiated fuel behaviour under reference accidents in order to check the adequacy of the current criteria, evaluate the safety margins, provide new technical bases for modelling, and allow an evolution of these criteria. Safety Assessment should be based on a well-thoughtout simulation/experimentation R\&D strategy, including inpile integral experiment. Technical Support Organisation (TSO), such as IRSN, should be leader in the investigation of accidental situations. They must also develop generic knowledge and computing tools because of the diversity of situations that will have to be assessed (technologies, operation, etc.). They have to get their own computing tools and methodologies in order to ensure the independence of their judgement and provide high-level skills training for expert assessment.

The IRSN is proposing an $\mathrm{R} \& \mathrm{D}$ programme, in the framework of international collaboration, in particular with TSOs, so as to be in a position to validate products, assessment methodologies, and operating conditions.

In this paper we focused on the $\mathrm{R} \& \mathrm{D}$ program proposed by IRSN in the context of a revision of acceptance criteria and the evolution of methodologies used for the safety assessment for LOCA and RIA.

In supporting evolution of LOCA criteria, IRSN is developing the CYCLADES program which adresses the main following topics.

(i) The analysis of cladding embrittlement with integral experiments taking into account bounding mechanical loads and the analysis of most appropriate parameters to characterise hydriring and oxidation of the cladding.

(ii) The axial fuel relocation, in developping experimental programs in Halden and CABRI reactors.

(iii) The coolability of flow blockage with a program of out-of-ile coolability experiments and single rod flow blockage experiments in CABRI reactors.

Concerning RIA, the IRSN strategy is based on mechanical tests on claddings, the forthcoming CIP and complementary programs on CABRI adressing postboiling crisis ruptures, fuel coolant interactions, and radiological releases.

All these programs are linked with a simulation strategy for extrapollation to reactor conditions.

\section{References}

[1] C. Grandjean, "A state of the art review of past programs devoted to fuel behaviour under LOCA conditions-part 1: clad swelling and rupture; assembly flow blockage," IRSN/DPAM/SEMCA 2005-313, http://net-science.irsn.org/. 
[2] C. Grandjean, "A state of the art review of past programs devoted to fuel behaviour under LOCA conditionspart 2: impact of clad swelling upon assembly cooling," IRSN/DPAM/SEMCA 2006-183, http://net-science.irsn.org/.

[3] C. Grandjean and G. Hache, "A state of the art review of past programs devoted to fuel behaviour under LOCA conditions_-part 3; cladding oxidation, resistance to quench and post quench loads," IRSN/DPAM/SEMCA 2008-093, http://net-science.irsn.org/.

[4] M. Billone, Y. Yan, T. Burtseva, and R. Daum, "Cladding embrittlement during postulated loss of coolant accidents," Tech. Rep. NUREG/CR-6967/ ANL-07/04, Argonne National Laboratory, Argonne, Ill, USA, 2007.

[5] M.C. Billone, Y. Yan, and T. Burtseva, "Overview of ANL LOCA programs," in Proceedings of the ANL LOCA Program Review Meeting, Argonne National Laboratory, Argonne, Ill, USA, October 2007.

[6] D. O. Hobson, "Ductile-brittle behaviour of Zry fuel cladding," in Proceedings of the Topical Meeting on Water Reactor Safety, Salt Lake City, Utah, USA, March 1973.

[7] R. E. Pawel, "Oxygen diffusion in beta zircaloy during steam oxidation," Journal of Nuclear Materials, vol. 50, no. 3, pp. 247 258, 1974.

[8] A. Sawatzky, "A proposed criterion for the oxygen embrittlement of Zircaloy 4 fuel cladding, zirconium in the nuclear industry," ASTM STP, vol. 681, pp. 479-496, 1979.

[9] H. M. Chung and T. F. Kassner, "Embritttlement criteria for Zircaloy Fuel Cladding applicable to accident situations in Light Water reactors," Summary Report, no. NUREG/CR-1344, ANL-7948, January 1980.

[10] J. C. Brachet, et al., "Overview of CEA analytical studies on the cladding behaviour during and after prototypical LOCA transients," in Proceedings of the OECD Ad-Hoc LOCA Meeting, Paris, France, June 2006.

[11] F. Nagase, M. Tanimoto, and H. Uetsuka, "Study on high burnup fuel behaviour under a LOCA condition at JAERI," in Proceedings of the IAEA Technical Committee Meeting on Fuel Behaviour under Transient and LOCA Conditions, Halden, Norway, September 2001.

[12] C. Grandjean and L. Belovski, "Oxidation of Zircaloy under LOCA conditions; development of the diffusion code DIFFOX 1.1," in Proceedings of the ANL LOCA Program Review Meeting, Argonne National Laboratory, Argonne, Ill, USA, October 2007.

[13] J. M. Broughton, et al., "PBF Loca tests," fuel behaviour report NUREG/CR-3184, EGG-2244, 1983.

[14] G. Repetto, F. Jacq, F. Barré, F. Lamare, and J. M. Ricaud, "DRACCAR, a new 3D thermal mechanical computer code to simulate LOCA transients on Nuclear Power Plants," in Proceedings of the International Congress on Advances in Nuclear Power Plants (ICAPP '09), Tokyo, Japan, May 2009.

[15] R. Landry, "Technical and regulatory basis for the reactivity Initiated accident interim acceptance criteria and guidance," Memorendum ML070220400, January 2007.

[16] L. O. Jernkvist, et al., "Assessment of core failure limits for LWRs under RIA," SKI Report 2005-16.

[17] M. T. Del Barrio and L. E. Herranz, "Failure criteria of a fuel rod during RIA,” CIEMAT Report DFN/SN-03/OP-02, 2002.

[18] J. Papin, et al., "Summary and interpretation of the CABRI REP-Na Program," Nuclear Technology, vol. 157, no. 3, pp. 230-250, 2007.

[19] J. Papin, M. Petit, C. Grandjean, and V. Georgenthum, "IRSN $\mathrm{R} \& \mathrm{D}$ studies in high burn up fuel behaviour under RIA and
LOCA conditions," in Proceedings of the Top Fuel Conference, Salamanca, Spain, October 2006.

[20] T. Fuketa, et al., "Behaviour of high burnup PWR fuels during simulated reactivity initiated accident conditions," in Proceedings of the Top Fuel Conference, Salamanca, Spain, October 2006.

[21] B. Cazalis, J. Desquines, C. Poussard, et al., "The PROMETRA program: fuel cladding mechanical behavior under high strain rate," Nuclear Technology, vol. 157, no. 3, pp. 215-229, 2007.

[22] V. Bessiron, "Modelling of clad-to-coolant heat transfer for RIA applications," Journal of Nuclear Science and Technology, vol. 44, no. 2, pp. 211-221, 2007.

[23] C. Sartoris, A. Taisne, M. Petit, F. Barré, and O. Marchand, "A consistent approach to assess safety criteria for reactivity initiated accidents," Nuclear Engineering and Design, vol. 240, no. 1, pp. 57-70, 2010.

[24] "Nuclear fuel behaviour during reactivity initiated accidents," in Proceedings of the OECD/NEA Workshop, Paris, France, September 2009. 

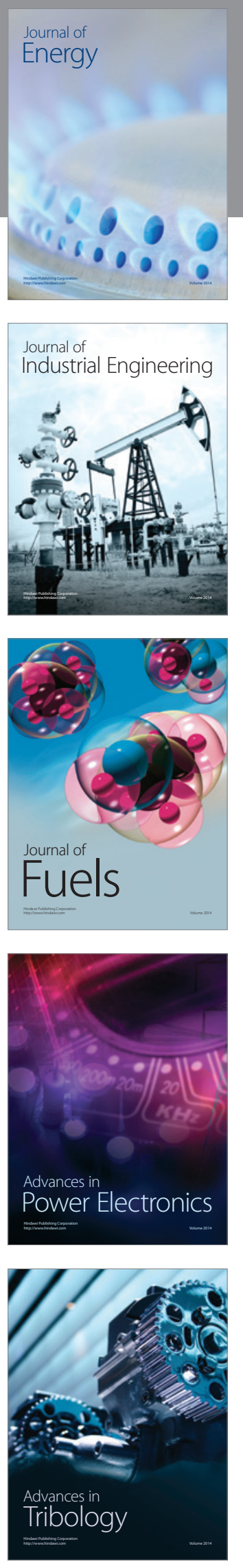
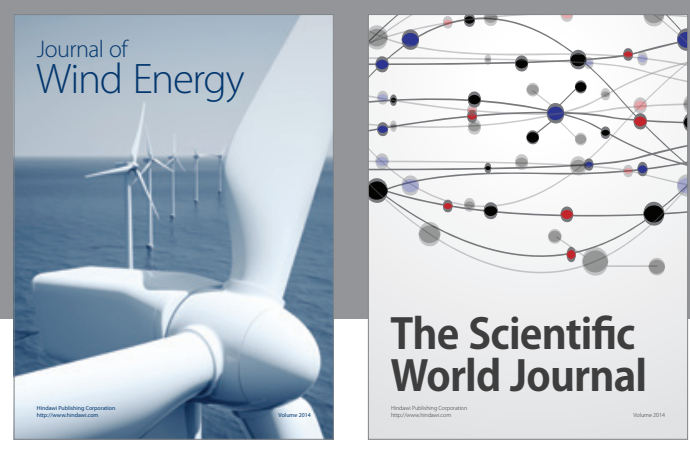

The Scientific World Journal

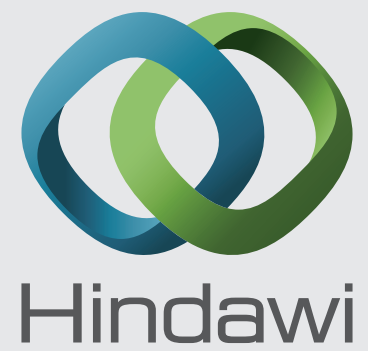

Submit your manuscripts at http://www.hindawi.com
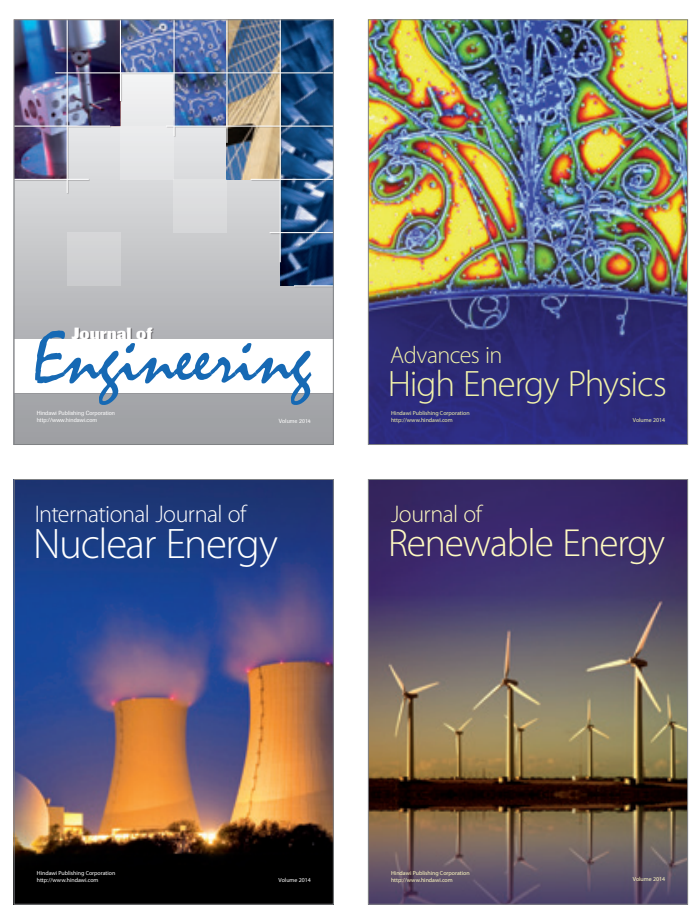

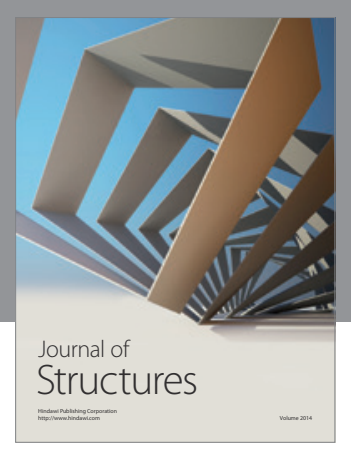

Rotating
Mechinery
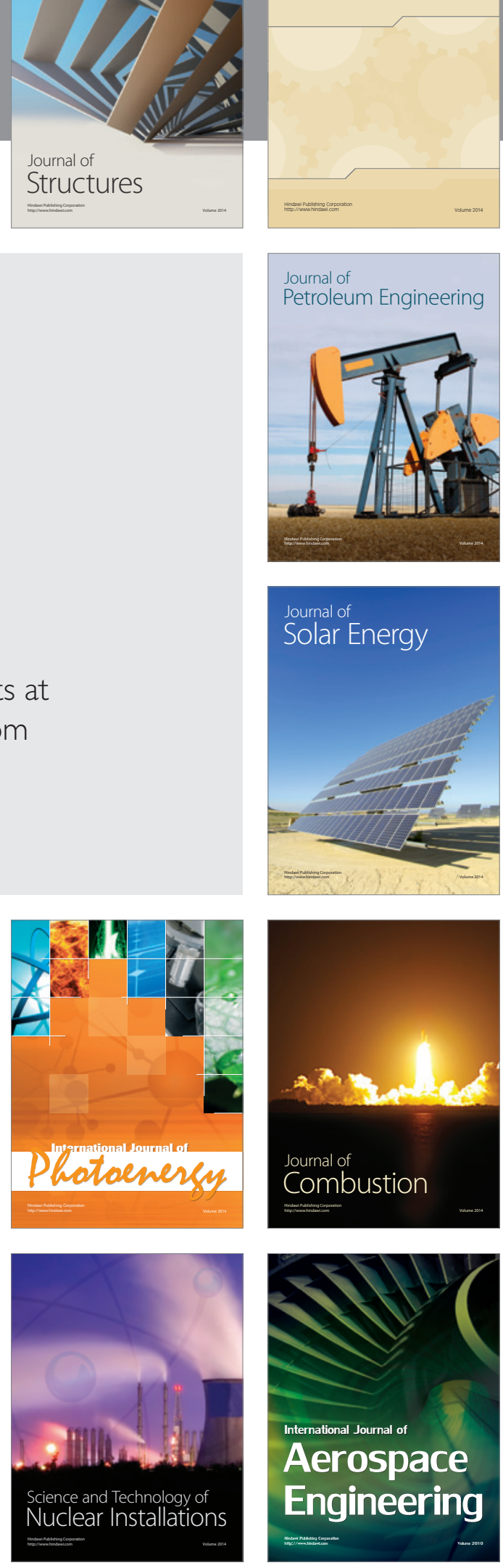